\title{
Meniscus Lateralis
}

National Cancer Institute

\section{Source}

National Cancer Institute. Meniscus Lateralis. NCI Thesaurus. Code C33097.

A semicircular meniscus located towards the outer aspect of the knee joint. 